\title{
An Operational Matrix Technique for Solving Variable Order Fractional Differential-Integral Equation Based on the Second Kind of Chebyshev Polynomials
}

\author{
Jianping Liu, Xia Li, and Limeng Wu \\ Hebei Normal University of Science and Technology, Qinhuangdao 066004, China \\ Correspondence should be addressed to Jianping Liu; liujianping0408@126.com
}

Received 12 March 2016; Accepted 11 May 2016

Academic Editor: Eugen Radu

Copyright (c) 2016 Jianping Liu et al. This is an open access article distributed under the Creative Commons Attribution License, which permits unrestricted use, distribution, and reproduction in any medium, provided the original work is properly cited.

\begin{abstract}
An operational matrix technique is proposed to solve variable order fractional differential-integral equation based on the second kind of Chebyshev polynomials in this paper. The differential operational matrix and integral operational matrix are derived based on the second kind of Chebyshev polynomials. Using two types of operational matrixes, the original equation is transformed into the arithmetic product of several dependent matrixes, which can be viewed as an algebraic system after adopting the collocation points. Further, numerical solution of original equation is obtained by solving the algebraic system. Finally, several examples show that the numerical algorithm is computationally efficient.
\end{abstract}

\section{Introduction}

Fractional differential equation (FDE) is an extension of integer-order model. Compared with the classical integerorder differential equation, FDE provides an excellent instrument for the description of memory and hereditary properties of various materials and processes. Therefore, it may be more accurate for modeling by FDE rather than integerorder differential equation. Researchers have pointed out that the fractional calculus plays an important role in modeling and many systems in interdisciplinary fields can be elegantly described with the help of fractional derivative, such as dynamics of earthquakes [1], viscoelastic systems [2], biological systems [3, 4], diffusion model [5], chaos [6], wave propagation [7], and partial bed-load transport [8].

Many literatures study the numerical algorithms for solving fractional calculus equations. Ahmadian et al. [9] proposed a computational method based on Jacobi polynomials for solving fuzzy linear FDE on interval $[0,1]$. Fu et al. [10] presented a Laplace transformed boundary particle method for numerical modeling of time fractional diffusion equations. Pang et al. [11] applied radial basis function meshless collocation method to the space-fractional advectiondispersion equations. Bhrawy and Zaky [12] analyzed an operational formulation based on Jacobi polynomials for time-space FDE with Dirichlet boundary conditions. Bhrawy et al. $[13,14]$ introduced a fractional integral operator matrix based on generalized Laguerre polynomials and shifted first of Chebyshev polynomials for solving fractional integral equations.

With further development of science research, more and more researchers have found that a variety of important dynamical problems exhibit fractional order behavior that may vary with time or space. This fact indicates that variable order calculus is a natural candidate to provide an effective mathematical framework for the description of complex dynamical problems. The modeling and application of variable order FDE have been a front subject. However, since the kernel of the variable order operators has a variable exponent, analytical solution of variable order FDE is usually difficult to obtain. The development of numerical algorithms to solve variable order FDE is necessary.

Only a few authors studied numerical methods of variable order FDE. Shen et al. [15] have given an approximate scheme for the variable order time fractional diffusion equation. Chen et al. $[16,17]$ paid their attention to Bernstein polynomials to solve variable order linear cable equation and variable order time fractional diffusion equation. An 
alternating direct method for the two-dimensional variable order fractional percolation equation was proposed in [18]. Explicit and implicit Euler approximations for FDE were introduced in [19]. A numerical method based on Legendre polynomials was presented for a class of variable order FDEs in [20]. Chen et al. [21] introduced the numerical solution for a class of nonlinear variable order FDEs with Legendre wavelets. In addition, for most literatures, they solved variable order FDE which is defined on the interval $[0,1]$. It is noteworthy that the Chebyshev polynomials family have beneficial properties so that they are widely used in approximation theory. However, the second kind of Chebyshev polynomials have been paid less attention for solving variable order FDE. Accordingly, we will solve a kind of variable order fractional differential-integral equations (FDIEs) defined on the interval $[0, R](R>0)$ based on the second kind of Chebyshev polynomials. The FDIE is shown as follows:

$$
\begin{aligned}
& D^{\alpha(t)} y(t)+a(t) \int_{0}^{t} y(z) d z+b(t) y^{\prime}(t)+c(t) y(t) \\
& =g(t), \quad y(0)=y_{0}, t \in[0, R],
\end{aligned}
$$

where $D^{\alpha(t)} y(t)$ is fractional derivative of $y(t)$ in Caputo's sense.

The basic idea of this approach is that we derive the differential operational matrix and the integral operational matrix based on Chebyshev polynomials. With the operational matrixes, (1) is transformed into the products of several dependent matrixes, which can be viewed as an algebraic system after taking the collocation points. By solving the algebraic system, the numerical solution of (1) is acquired. Since the second kind of Chebyshev polynomials are orthogonal to each other, the operational matrixes based on Chebyshev polynomials greatly reduce the size of computational work while accurately providing the series solution. From some numerical examples, we can see that our results are in good agreement with exact solution. Numerical result demonstrates the validity of this algorithm.

The paper is organized as follows. In Section 2, some necessary preliminaries are introduced. In Section 3, the basic definition and property of the second kind of Chebyshev polynomials are given. In Section 4, function approximation is given. In Section 5, two kinds of operational matrixes are derived and we applied the operational matrixes to solve the equation as given at the beginning. In Section 6, we present some numerical examples to demonstrate the efficiency of the algorithm. We end the paper with a few concluding remarks in Section 7.

\section{Preliminaries}

There are several definitions for variable order fractional derivatives, such as the one in Riemann-Liouville's sense and the one in Caputo's sense [22]. In this paper, the definition in Caputo's sense is considered.
Definition 1. Caputo fractional derivate with order $\alpha(t)$ is defined by

$$
\begin{aligned}
D^{\alpha(t)} y(t)= & \frac{1}{\Gamma(1-\alpha(t))} \int_{0_{+}}^{t}(t-\tau)^{-\alpha(t)} y^{\prime}(\tau) d \tau \\
& +\frac{y(0+)-y(0-)}{\Gamma(1-\alpha(t))} t^{-\alpha(t)} .
\end{aligned}
$$

If we assume the starting time in a perfect situation, we can get Definition 2 as follows.

Definition 2. Consider the following:

$$
D^{\alpha(t)} y(t)=\frac{1}{\Gamma(1-\alpha(t))} \int_{0}^{t}(t-\tau)^{-\alpha(t)} y^{\prime}(\tau) d \tau .
$$

By Definition 2, we can get the following formula [16]:

$$
D^{\alpha(t)} t^{n}= \begin{cases}\frac{\Gamma(n+1)}{\Gamma(n+1-\alpha(t))} t^{n-\alpha(t)}, & n=1,2, \ldots, \\ 0, & n=0 .\end{cases}
$$

\section{The Shifted Second Kind of Chebyshev Polynomials}

The second kind of Chebyshev polynomials are defined on the interval $I=[-1,1]$. They are orthogonal to each other with respect to the weight function $\omega(x)=\sqrt{1-x^{2}}$. They satisfy the following formulas:

$$
\begin{aligned}
U_{0}(x) & =1, \\
U_{1}(x) & =2 x, \\
U_{n+1}(x) & =2 x U_{n}(x)-U_{n-1}(x), \\
& n=1,2, \ldots, \\
\int_{-1}^{1} \sqrt{1-x^{2}} U_{n}(x) U_{m}(x) d x & = \begin{cases}\frac{\pi}{2}, & m=n, \\
0, & m \neq n .\end{cases}
\end{aligned}
$$

For $t \in[0, R]$, let $x=2 t / R-1$. We can get shifted second kind of Chebyshev polynomials $\widetilde{U}_{n}(t)=U_{n}(2 t / R-1)$, which are also orthogonal with respect to weight function $\omega(t)=$ $\sqrt{t R-t^{2}}$ for $t \in[0, R]$. They satisfy the following formulas:

$$
\begin{aligned}
& \widetilde{U}_{0}(t)=1, \\
& \widetilde{U}_{1}(t)=\frac{4 t}{R}-2, \\
& \widetilde{U}_{n+1}(t)=2\left(\frac{2 t}{R}-1\right) \widetilde{U}_{n}(t)-\widetilde{U}_{n-1}(t),
\end{aligned}
$$

$$
n=1,2, \ldots \text {, }
$$

$$
\int_{0}^{R} \sqrt{t R-t^{2}} \widetilde{U}_{n}(t) \widetilde{U}_{m}(t) d t= \begin{cases}\frac{\pi}{8} R^{2}, & m=n, \\ 0, & m \neq n .\end{cases}
$$


The shifted second kind of Chebyshev polynomials can also be expressed as

$$
\begin{aligned}
& \widetilde{U}_{n}(t) \\
&= \begin{cases}1, & n=0, \\
\sum_{k=0}^{[n / 2]}(-1)^{k} \frac{(n-k) !}{k !(n-2 k) !}\left(\frac{4 t}{R}-2\right)^{n-2 k}, & n \geq 1,\end{cases}
\end{aligned}
$$

where $[n / 2]$ denotes the maximum integer which is no more than $n / 2$.

Let $\Psi_{n}(t)=\left[\widetilde{U}_{0}(t), \widetilde{U}_{1}(t), \ldots, \widetilde{U}_{n}(t)\right]^{T}$ and $\mathbf{T}_{n}(t)=$ $\left[1, t, \ldots, t^{n}\right]^{T}$. Therefore,

$$
\Psi_{n}(t)=\mathbf{A T}_{n}(t),
$$

where

$$
\begin{aligned}
\mathbf{A} & =\mathbf{B C}, \\
\mathbf{C} & =\left[\begin{array}{ccccc}
1 & 0 & 0 & \cdots & 0 \\
C_{1}^{0}\left(-\frac{R}{2}\right)^{1-0} & C_{1}^{1}\left(-\frac{R}{2}\right)^{1-1} & 0 & \cdots & 0 \\
C_{2}^{0}\left(-\frac{R}{2}\right)^{2-0} & C_{2}^{1}\left(-\frac{R}{2}\right)^{2-1} & C_{2}^{2}\left(-\frac{R}{2}\right)^{2-2} & \cdots & 0 \\
\vdots & \vdots & \vdots & \vdots & \vdots \\
C_{n}^{0}\left(-\frac{R}{2}\right)^{n-0} & C_{n}^{1}\left(-\frac{R}{2}\right)^{n-1} & C_{n}^{2}\left(-\frac{R}{2}\right)^{n-2} & \cdots & C_{n}^{n}\left(-\frac{R}{2}\right)^{n-n}
\end{array}\right] .
\end{aligned}
$$

If $n$ is an even number, then

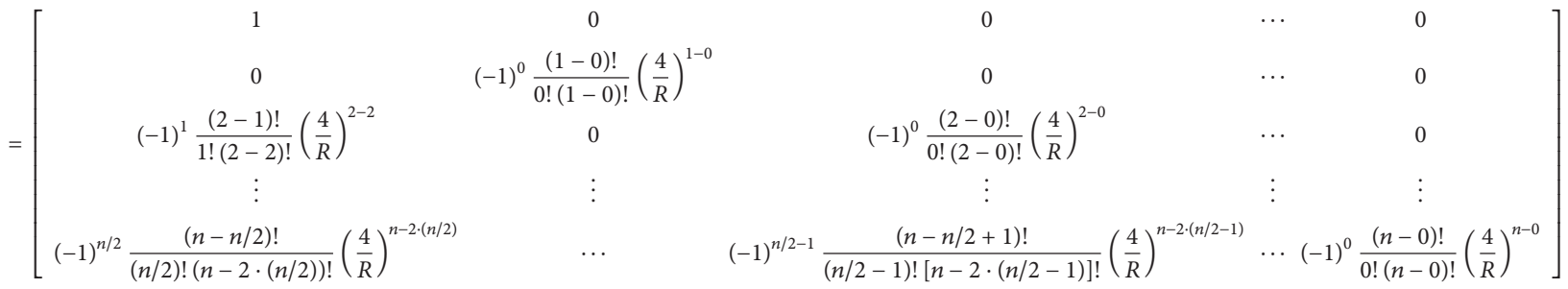

If $n$ is an odd number, then

B

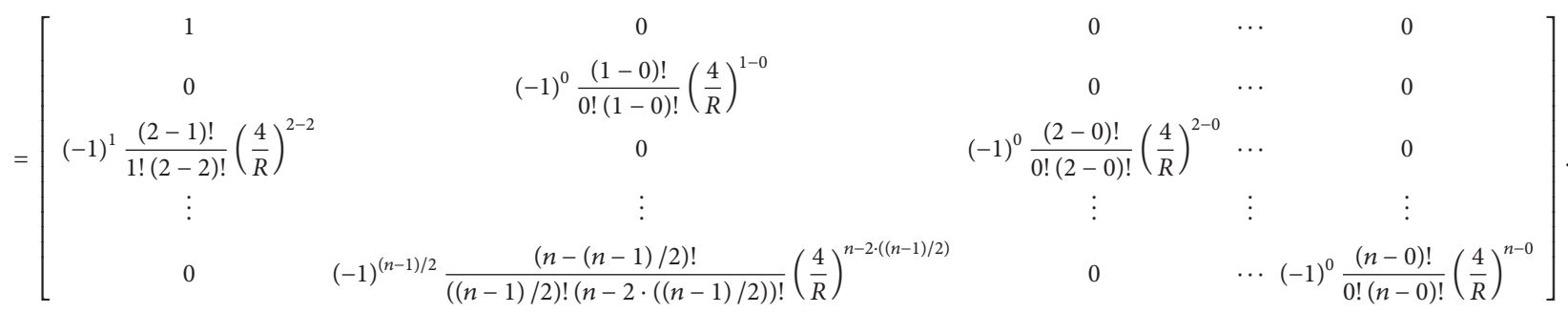

Therefore, we can easily gain (12) as follows:

$$
\mathbf{T}_{n}(t)=\mathbf{A}^{-1} \Psi_{n}(t)
$$

\section{Function Approximation}

Theorem 3. Suppose that the function $y(t)$ is $n+1$ times continuously differentiable on the interval $[0, R]$. Let $u_{n}(t)=$ 
$\sum_{i=0}^{n} \lambda_{i} \widetilde{U}_{i}(t)=\Lambda^{T} \Psi_{n}(t)$ be the best square approximation function of $y(t)$, where $\Lambda=\left[\lambda_{0}, \lambda_{1}, \ldots, \lambda_{n}\right]^{T}$. Then,

$$
\left\|y(t)-u_{n}(t)\right\|_{2} \leq \frac{M S^{n+1} R}{(n+1) !} \sqrt{\frac{\pi}{8}},
$$

where $M=\max _{t \in[0, R]} y^{n+1}(t)$ and $S=\max \left\{R-t_{0}, t_{0}\right\}$.

Proof. We consider the Taylor polynomials:

$$
\begin{aligned}
& y(t)=y\left(t_{0}\right)+y^{\prime}\left(t_{0}\right)\left(t-t_{0}\right)+\cdots \\
& \qquad \begin{aligned}
& \\
& +y^{(n)}\left(t_{0}\right) \frac{\left(t-t_{0}\right)^{n}}{n !}+y^{(n+1)}(\eta) \frac{\left(t-t_{0}\right)^{n+1}}{(n+1) !}, \\
& t_{0} \in[0, R],
\end{aligned}
\end{aligned}
$$

where $\eta$ is between $t$ and $t_{0}$.

Let

$$
\begin{aligned}
p_{n}(t)= & y\left(t_{0}\right)+y^{\prime}\left(t_{0}\right)\left(t-t_{0}\right)+\cdots \\
& +y^{(n)}\left(t_{0}\right) \frac{\left(t-t_{0}\right)^{n}}{n !} .
\end{aligned}
$$

Then,

$$
\left|y(t)-p_{n}(t)\right|=\left|y^{(n+1)}(\eta) \frac{\left(t-t_{0}\right)^{n+1}}{(n+1) !}\right|
$$

Since $u_{n}(t)=\sum_{i=0}^{n} \lambda_{i} \widetilde{U}_{i}(t)=\Lambda^{T} \Psi_{n}(t)$ is the best square approximation function of $y(t)$, we can gain

$$
\begin{aligned}
& \left\|y(t)-u_{n}(t)\right\|_{2}^{2} \leq\left\|y(t)-p_{n}(t)\right\|_{2}^{2} \\
& =\int_{0}^{R} \omega(t)\left[y(t)-p_{n}(t)\right]^{2} d t \\
& =\int_{0}^{R} \omega(t)\left[y^{(n+1)}(\eta) \frac{\left(t-t_{0}\right)^{n+1}}{(n+1) !}\right]^{2} d t \\
& \leq \frac{M^{2}}{[(n+1) !]^{2}} \int_{0}^{R} \omega(t)\left(t-t_{0}\right)^{2 n+2} d t \\
& =\frac{M^{2}}{[(n+1) !]^{2}} \int_{0}^{R} \sqrt{t R-t^{2}}\left(t-t_{0}\right)^{2 n+2} d t .
\end{aligned}
$$

Let $S=\max \left\{R-t_{0}, t_{0}\right\}$. Therefore,

$$
\begin{aligned}
\left\|y(t)-u_{n}(t)\right\|_{2}^{2} & \leq \frac{M^{2} S^{2 n+2}}{[(n+1) !]^{2}} \int_{0}^{R} \sqrt{t R-t^{2}} d t \\
& =\frac{M^{2} S^{2 n+2} R^{2}}{(n+1) !^{2}} \frac{\pi}{8} .
\end{aligned}
$$

Finally, by taking the square roots, Theorem 3 can be proved.

\section{Operational Matrix Technique for Solving Variable Order Fractional Differential-Integral Equation}

5.1. Differential Operational Matrix of $D^{\alpha(t)} \Psi_{n}(t)$. Considering (8), we obtain

$$
\begin{aligned}
D^{\alpha(t)} \Psi_{n}(t) & =D^{\alpha(t)}\left(\mathbf{A T}_{n}(t)\right) \\
& =\mathbf{A} D^{\alpha(t)}\left(\left[1, t, \ldots, t^{n}\right]^{T}\right) .
\end{aligned}
$$

Then, according to (4), we can get

$$
\begin{aligned}
& D^{\alpha(t)}\left(\left[1, t, \ldots, t^{n}\right]^{T}\right) \\
& =\left[0, \frac{\Gamma(2)}{\Gamma(2-\alpha(t))} t^{1-\alpha(t)}, \ldots, \frac{\Gamma(n+1)}{\Gamma(n+1-\alpha(t))} t^{n-\alpha(t)}\right]^{T} \\
& =\left[\begin{array}{cccc}
0 & 0 & \ldots & 0 \\
0 & \frac{\Gamma(2)}{\Gamma(2-\alpha(t))} t^{-\alpha(t)} & \ldots & 0 \\
\vdots & \vdots & \vdots & \vdots \\
0 & 0 & \ldots & \frac{\Gamma(n+1)}{\Gamma(n+1-\alpha(t))} t^{-\alpha(t)}
\end{array}\right]\left[\begin{array}{c}
1 \\
t \\
\vdots \\
t^{n}
\end{array}\right] \\
& =\mathbf{M}_{\alpha(t)} \mathbf{A}^{-1} \Psi_{n}(t),
\end{aligned}
$$

where

$$
\begin{aligned}
& \mathbf{M}_{\alpha(t)} \\
& =\left[\begin{array}{cccc}
0 & 0 & \cdots & 0 \\
0 & \frac{\Gamma(2)}{\Gamma(2-\alpha(t))} t^{-\alpha(t)} & \cdots & 0 \\
\vdots & \vdots & \vdots & \vdots \\
0 & 0 & \cdots & \frac{\Gamma(n+1)}{\Gamma(n+1-\alpha(t))} t^{-\alpha(t)}
\end{array}\right] .
\end{aligned}
$$

Therefore,

$$
D^{\alpha(t)} \Psi_{n}(t)=\mathbf{A M}_{\alpha(t)} \mathbf{A}^{-1} \Psi_{n}(t) .
$$

$\mathbf{A M}_{\alpha(t)} \mathbf{A}^{-1}$ is called the operational matrix of $D^{\alpha(t)} \Psi_{n}(t)$. In particular, for $\alpha(t)=1$, we can get

$$
\Psi_{n}^{\prime}(t)=\mathbf{A M}_{1} \mathbf{A}^{-1} \Psi_{n}(t) .
$$

5.2. Integral Operational Matrix of $\int_{0}^{t} \Psi_{n}(z) d z$. Let $\int_{0}^{t} \Psi_{n}(z) d z \approx \mathbf{P} \Psi_{n}(t)$. $\mathbf{P}$ is called the integral operational matrix. The objective of this section is to generate this matrix P. 
According to (8), we have

$$
\begin{aligned}
& \int_{0}^{t} \Psi_{n}(z) d z=\int_{0}^{t} \mathbf{A} \mathbf{T}_{n}(z) d z=\mathbf{A} \int_{0}^{t} \mathbf{T}_{n}(z) d z \\
&=\mathbf{A} \int_{0}^{t}\left[1, z, \ldots, z^{n}\right]^{T} d z \\
&=\mathbf{A}\left[\begin{array}{c}
t \\
\frac{1}{2} t^{2} \\
\vdots \\
\frac{1}{n+1} t^{n+1}
\end{array}\right] \\
&=\mathbf{A}\left[\begin{array}{cccc}
1 & 0 & \cdots & 0 \\
0 & \frac{1}{2} & \cdots & 0 \\
\vdots & \vdots & \vdots & \vdots \\
0 & 0 & \cdots & \frac{1}{n+1}
\end{array}\right]\left[\begin{array}{c}
t \\
t^{2} \\
\vdots \\
t^{n+1}
\end{array}\right] \\
&=\mathbf{A}_{p} \mathbf{T}_{p}, \quad \ldots \\
&
\end{aligned}
$$

where

$$
\begin{gathered}
\mathbf{A}_{p}=\mathbf{A}\left[\begin{array}{cccc}
1 & 0 & \cdots & 0 \\
0 & \frac{1}{2} & \cdots & 0 \\
\vdots & \vdots & \vdots & \vdots \\
0 & 0 & \cdots & \frac{1}{n+1}
\end{array}\right], \\
\mathbf{T}_{p}=\left[\begin{array}{c}
t \\
t^{2} \\
\vdots \\
t^{n+1}
\end{array}\right] .
\end{gathered}
$$

According to (8), we can get

$$
t^{k}=A_{[k+1]}^{-1} \Psi_{n}(t), \quad k=1, \ldots, n,
$$

where $A_{[k]}^{-1}$ is $k$ row of $A^{-1}$.

We approximate $t^{n+1}$ by the second kind of Chebyshev polynomials as follows [22]:

$$
t^{n+1} \approx \mathbf{c}_{n+1}^{T} \Psi_{n}(t),
$$

where

$$
\begin{aligned}
\mathbf{c}_{n+1} & =\frac{8}{\pi R^{2}}\left(t^{n+1}, \Psi_{n}(t)\right) \\
& =\frac{8}{\pi R^{2}} \int_{0}^{R} \sqrt{t R-t^{2}} t^{n+1} \Psi_{n}(t) d t .
\end{aligned}
$$

Accordingly,

$$
\begin{aligned}
& \mathbf{P}_{1}=\left[\begin{array}{c}
A_{[2]}^{-1} \\
A_{[3]}^{-1} \\
\vdots \\
A_{[n+1]}^{-1} \\
\mathbf{c}_{n+1}^{T}
\end{array}\right], \\
& \mathbf{T}_{p} \approx \mathbf{P}_{1} \Psi_{n}(t) .
\end{aligned}
$$

Therefore,

$$
\begin{gathered}
\mathbf{P}=\mathbf{A}_{p} \mathbf{P}_{1}, \\
\int_{0}^{t} \Psi_{n}(z) d z \approx \mathbf{A}_{p} \mathbf{P}_{1} \Psi_{n}(t) .
\end{gathered}
$$

5.3. Solve Variable Order Fractional Differential-Integral Equation by Operational Matrix Technique. Let $y(t) \approx u_{n}(t)=$ $\sum_{i=0}^{n} \lambda_{i} \widetilde{U}_{i}(t)=\Lambda^{T} \Psi_{n}(t)$. According to (22), (23), and (31), original equation (1) is transformed into (32) as follows:

$$
\begin{aligned}
\Lambda^{T} \mathbf{A M}_{\alpha(t)} \mathbf{A}^{-1} \Psi_{n}(t)+a(t) \Lambda^{T} \mathbf{P} \Psi_{n}(t) & \\
& +b(t) \Lambda^{T} \mathbf{A} \mathbf{M}_{1} \mathbf{A}^{-1} \Psi_{n}(t)+c(t) \Lambda^{T} \Psi_{n}(t) \\
= & g(t), \quad t \in[0, R] .
\end{aligned}
$$

Taking the collocation points $t_{i}=R((2 i+1) / 2(n+1)), \quad i=$ $0,1, \ldots, n$, to process $(32)$, we can gain

$$
\begin{aligned}
\Lambda^{T} & \mathbf{A M}_{\alpha\left(t_{i}\right)} \mathbf{A}^{-1} \Psi_{n}\left(t_{i}\right)+a\left(t_{i}\right) \Lambda^{T} \mathbf{P} \Psi_{n}\left(t_{i}\right) \\
& +b\left(t_{i}\right) \Lambda^{T} \mathbf{A} \mathbf{M}_{1} \mathbf{A}^{-1} \Psi_{n}\left(t_{i}\right)+c\left(t_{i}\right) \Lambda^{T} \Psi_{n}\left(t_{i}\right) \\
& =g\left(t_{i}\right) .
\end{aligned}
$$

Solving algebraic system (33) by Newton method [22], we can gain the vector $\Lambda=\left[\lambda_{0}, \lambda_{1}, \ldots, \lambda_{n}\right]^{T}$. Subsequently, numerical solution $u_{n}(t)=\Lambda^{T} \Psi_{n}(t)$ is obtained.

\section{Numerical Examples and Result Analysis}

In this section, we verify the efficiency of operational matrix technique to support the above theoretical discussion. We compare the numerical solution with the exact solution by using our approach. The results indicate that our algorithm is a powerful tool for solving variable order FDIE. In this section, the notation

$$
\varepsilon=\max _{i=0,1, \ldots, n}\left|y\left(t_{i}\right)-u_{n}\left(t_{i}\right)\right|
$$

is used to show the precision of our proposed algorithm, where $t_{i}=R((2 i+1) / 2(n+1)), i=0,1, \ldots, n$. 
TABLE 1: Computational results of Example 1 for $R=1$ and $n=3$.

\begin{tabular}{lcccc}
\hline$t$ & $\Lambda$ & Exact solution & Numerical solution & Absolute error \\
\hline 0.1250 & 9.0625 & 1.9531 & 1.9531 & $0.3952 e-13$ \\
0.3750 & 5.0000 & 6.3281 & 6.3281 & $0.2220 e-13$ \\
0.6250 & 0.3125 & 11.3281 & 11.3281 & $0.0711 e-13$ \\
0.8750 & 0.0000 & 16.9531 & 16.9531 & $0.0355 e-13$ \\
\hline
\end{tabular}

TABLE 2: Computational results of Example 1 for $R=1$ and $n=4$.

\begin{tabular}{lccrr}
\hline$t$ & $\Lambda$ & Exact solution & Numerical solution & Absolute error \\
\hline 0.1000 & 9.0625 & 1.5500 & 1.5500 & $0.3524 e-12$ \\
0.3000 & 5.0000 & 4.9500 & 4.9500 & $0.2336 e-12$ \\
0.5000 & 0.3125 & 8.7500 & 8.7500 & $0.1350 e-12$ \\
0.7000 & 0.0000 & 12.9500 & 12.9500 & $0.0480 e-12$ \\
0.9000 & -0.0000 & 17.5500 & 17.5500 & $0.0249 e-12$ \\
\hline
\end{tabular}

TABLE 3: Error $\varepsilon$ of Example 1 for different $R$.

\begin{tabular}{lcccc}
\hline$R$ & $n=3$ & $n=4$ & $n=5$ & $n=6$ \\
\hline$R=2$ & $1.7408 e-13$ & $5.2847 e-13$ & $6.9678 e-13$ & $5.2793 e-12$ \\
$R=4$ & $2.6148 e-12$ & $8.7255 e-12$ & $7.5460 e-12$ & $2.6716 e-11$ \\
$R=8$ & $1.3301 e-11$ & $4.0711 e-10$ & $3.6262 e-09$ & $2.4797 e-09$ \\
\hline
\end{tabular}

Example 1. Consider the following nonlinear variable order FDIE:

$$
\begin{array}{r}
D^{\alpha(t)} y(t)+6 \int_{0}^{t} y(z) d z+2 t y^{\prime}(t)+y(t)=g(t), \\
y(0)=0, t \in[0, R],
\end{array}
$$

where

$$
\begin{aligned}
\alpha(t)= & \frac{3(\sin t+\cos t)}{5} \\
g(t)= & \frac{10}{\Gamma(3-\alpha(t))} t^{2-\alpha(t)}+\frac{15}{\Gamma(2-\alpha(t))} t^{1-\alpha(t)} \\
& +10 t^{3}+70 t^{2}+45 t
\end{aligned}
$$

The exact solution is $y(t)=5 t^{2}+15 t$. We find the numerical solution of Example 1 in MATLAB 2012 by our technique.

The computational results are shown in Tables $1-3$. As seen from Tables 1 and 2 , the vector $\Lambda=\left[\lambda_{0}, \lambda_{1}, \ldots, \lambda_{n}\right]^{T}$ is mainly composed of three terms, namely, $\lambda_{0}=9.0625, \lambda_{1}=$ 5.0000 , and $\lambda_{2}=0.3125$. It is evident that the numerical solution converges to the exact solution. Only a small number of the second Chebyshev polynomials are needed to reach high precision, which verifies the correction and high efficiency of our algorithm. In addition, for $R=1$, with $n$ increasing, absolute errors of $n=3$ are a little bigger than those of $n=4$ because of round-off error in MATLAB. Therefore, the approximation effect of $n=3$ is better than that of $n=4$. Furthermore, our algorithm can obtain the solution not only for $R=1$ but also for $R>1$. We extend the interval from
$[0,1]$ to $\left[0,2^{k}\right], k=1,2,3$. Similarly, we also get the perfect results shown in Table 3.

In Table 3 , we list $\varepsilon$ for different $R$ and $n$. Also, for the same $R$, with $n$ increasing, $\varepsilon$ is generally a little bigger because of round-off error in MATLAB. On the other hand, for the same $n$, with $R$ increasing, $\varepsilon$ becomes slightly bigger, which is consistent with Theorem 3. However, every error $\varepsilon$ is small enough to meet the practical engineering application. Figure 1 also shows that the numerical solutions are very close to exact solutions. It verifies the correction and efficiency of the algorithm in this study.

Example 2. Consider another nonlinear variable order FDIE:

$$
\begin{aligned}
& D^{t / 3} y(t)+e^{t} \int_{0}^{t} y(z) d z+\sin t y^{\prime}(t)+(t-1) y(t) \\
& \quad=g(t), \quad y(0)=1, t \in[0, R]
\end{aligned}
$$

where

$$
\begin{aligned}
g(t)= & \frac{3 t^{1-t / 3}(7 t-6)}{\left(18-9 t+t^{2}\right) \Gamma(1-t / 3)}+e^{t}\left(t-\frac{t^{2}}{2}+\frac{t^{3}}{3}\right) \\
& +(2 t-1) \sin t+(t-1)\left(t^{2}-t+1\right)
\end{aligned}
$$

The exact solution is $y(t)=t^{2}-t+1$. We still obtain the solution as Example 1 by using our algorithm. The computational results are shown in Tables 4-6. As seen from Tables 4 and 5 , the vector $\Lambda=\left[\lambda_{0}, \lambda_{1}, \ldots, \lambda_{n}\right]^{T}$ is mainly composed of two terms, namely, $\lambda_{0}=0.8125$ and $\lambda_{2}=$ 0.0625 . It is evident that the numerical solution converges to the exact solution. In particular, for $R=1$ and $n=3$, every absolute error at the collocation point is 0 because of roundoff in MATLAB. Therefore, the approximation effect of $n=3$ is better than that of $n=4$.

Finally, we extend the interval from $[0,1]$ to $[0, R], R=$ 2,3 . Similarly, we also get the perfect results as shown in Figure 2 and Table 6 . Figure 2 shows the exact solution and numerical solution for different $R$ at collocation points, which demonstrates that the numerical solution is very close to 
TABLE 4: Computational results of Example 2 for $R=1$ and $n=3$.

\begin{tabular}{lcccc}
\hline$t$ & $\Lambda$ & Exact solution & Numerical solution & Absolute error \\
\hline 0.1250 & 0.8125 & 0.8906 & 0.8906 & 0 \\
0.3750 & 0.0000 & 0.7656 & 0.7656 & 0 \\
0.6250 & 0.0625 & 0.7656 & 0.7656 & 0 \\
0.8750 & 0.0000 & 0.8906 & 0.8906 & 0 \\
\hline
\end{tabular}

TABLE 5: Computational results of Example 2 for $R=1$ and $n=4$.

\begin{tabular}{lcccc}
\hline$t$ & $\Lambda$ & Exact solution & Numerical solution & Absolute error \\
\hline 0.1000 & 0.8125 & 0.9100 & 0.9100 & $0.1110 e-15$ \\
0.3000 & -0.0000 & 0.7900 & 0.7900 & $0.2220 e-15$ \\
0.5000 & 0.0625 & 0.7500 & 0.7500 & $0.5551 e-15$ \\
0.7000 & 0.0000 & 0.7900 & 0.7900 & $0.4441 e-15$ \\
0.9000 & 0.0000 & 0.9100 & 0.9100 & $0.6661 e-15$ \\
\hline
\end{tabular}

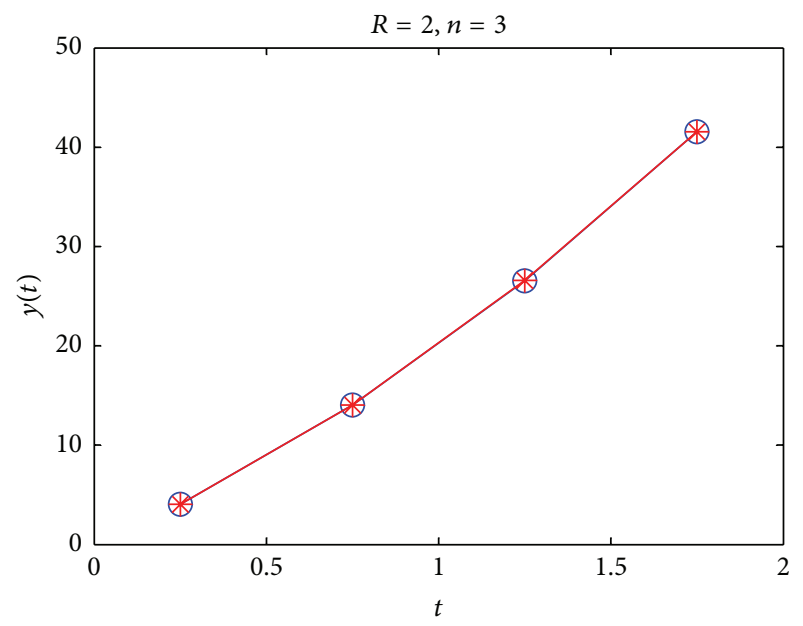

$\bigcirc$ Exact solution

* Numerical solution

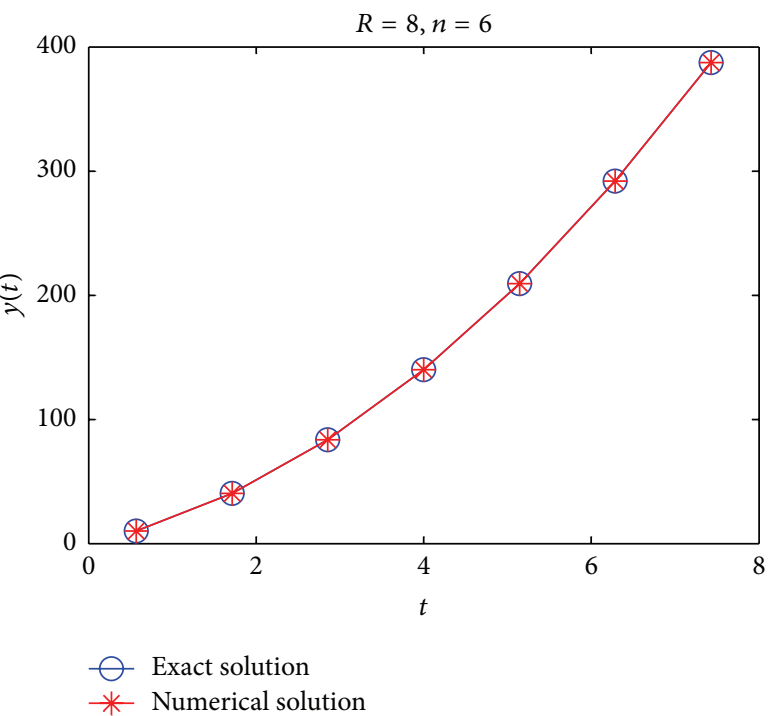

* Numerical solution

FIgURE 1: Exact solution and numerical solution of Example 1.
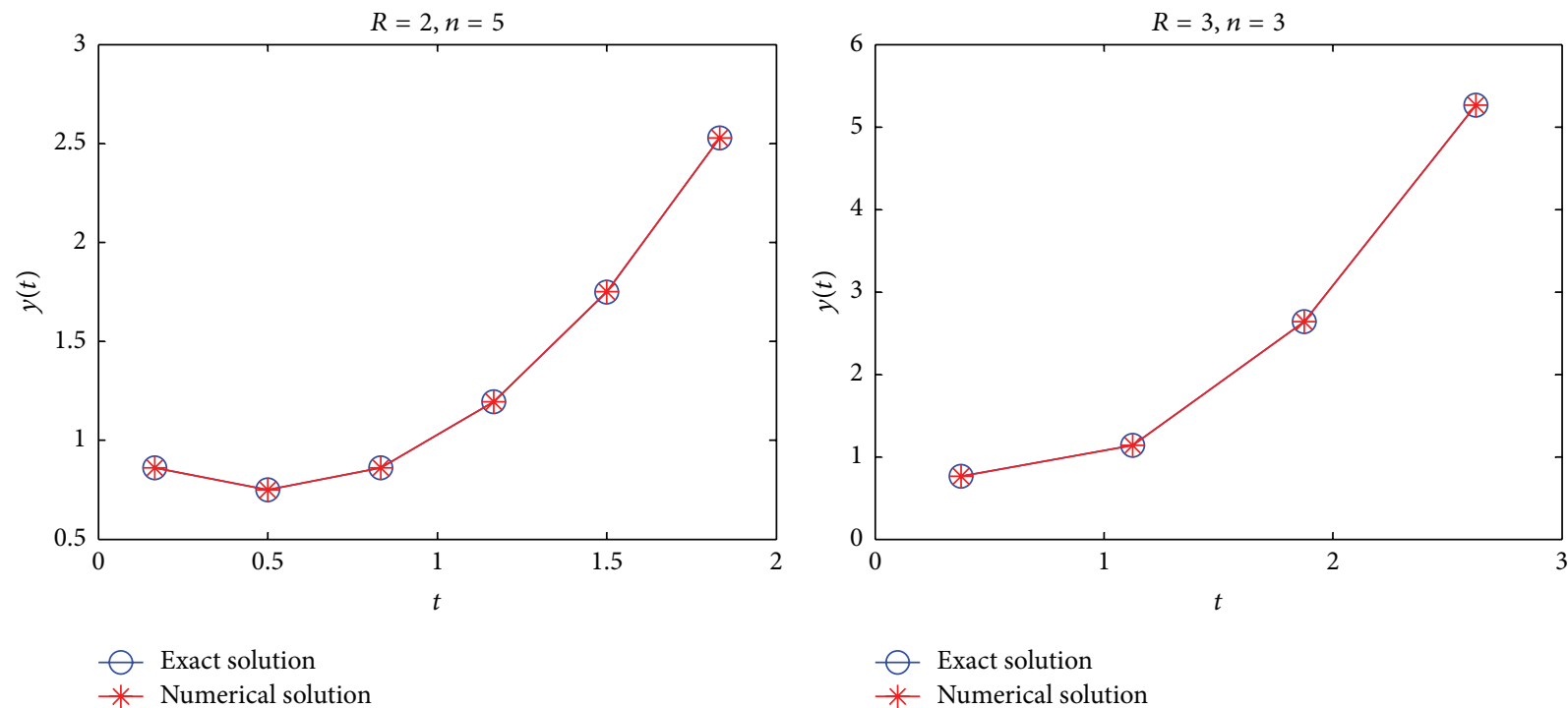

* Numerical solution

FIgURE 2: Exact solution and numerical solution of Example 2. 
TABLE 6: Error $\varepsilon$ of Example 2 for different $R$.

\begin{tabular}{lcccc}
\hline$R$ & $n=3$ & $n=4$ & $n=5$ & $n=6$ \\
\hline$R=2$ & $4.8850 e-15$ & $6.5503 e-15$ & $1.6986 e-14$ & $4.2055 e-13$ \\
$R=3$ & $6.1173 e-14$ & $2.8866 e-14$ & $2.9576 e-13$ & $3.8725 e-13$
\end{tabular}

the exact solution. From Table 6 , for the same $R$, with $n$ increasing, $\varepsilon$ is generally a little bigger because of round-off error. From another point of view, for the same $n$, with $R$ increasing, $\varepsilon$ becomes slightly bigger, which is consistent with Theorem 3 . There is the same trend for the other $R$. Every error $\varepsilon$ is small enough to meet the practical engineering application.

\section{Conclusions}

In this paper, we present a numerical technique for solving the variable order FDIE based on the second kind of Chebyshev polynomials. Taking advantage of the definition of the variable order fraction derivative and the simplicity of the second kind of Chebyshev polynomials, we transform the FDIE into an algebraic system. By solving the algebraic system, the numerical solution is acquired. Numerical examples show that the numerical solution is in very good accordance with the exact solution. The technique can be applied to solve the other variable order fractional differential problems.

\section{Competing Interests}

The authors declare that they have no competing interests.

\section{Acknowledgments}

This work is funded in part by the Natural Science Foundation of Hebei Province (no. A2015407063), China, and is funded in part by the Teaching Research Project of Hebei Normal University of Science and Technology (no. JYZD201413), China. The work is also funded by Scientific Research Foundation of Hebei Normal University of Science and Technology.

\section{References}

[1] A. A. Kilbas, H. M. Srivastava, and J. J. Trujillo, Theory and Application of Fractional Differential Equations, vol. 204, Elsevier Science B.V., New York, NY, USA, 2006.

[2] Y. A. Rossikhin and M. V. Shitikova, "Application of fractional derivatives to the analysis of damped vibrations of viscoelastic single mass systems," Acta Mechanica, vol. 120, no. 1, pp. 109$125,1997$.

[3] E. Ahmed and A. S. Elgazzar, "On fractional order differential equations model for nonlocal epidemics," Physica A: Statistical Mechanics and its Applications, vol. 379, no. 2, pp. 607-614, 2007.

[4] N. Zalp and E. Demirci, "A fractional order SEIR model with vertical transmission," Mathematical and Computer Modelling, vol. 54, no. 1-2, pp. 1-6, 2011.
[5] H. G. Sun, W. Chen, and Y. Q. Chen, "Variable-order fractional differential operators in anomalous diffusion modeling," Physica A: Statistical Mechanics and Its Applications, vol. 388, no. 21, pp. 4586-4592, 2009.

[6] W. Chen, "A speculative study of 2/3-order fractional Laplacian modeling of turbulence: some thoughts and conjectures," Chaos: An Interdisciplinary Journal of Nonlinear Science, vol. 16, no. 2, Article ID 023126, 2006.

[7] W. Chen, H. Sun, X. Zhang, and D. Korošak, "Anomalous diffusion modeling by fractal and fractional derivatives," Computers \& Mathematics with Applications, vol. 59, no. 5, pp. 1754-1758, 2010.

[8] H. G. Sun, D. Chen, Y. Zhang, and L. Chen, "Understanding partial bed-load transport: experiments and stochastic model analysis," Journal of Hydrology, vol. 521, pp. 196-204, 2015.

[9] A. Ahmadian, M. Suleiman, S. Salahshour, and D. Baleanu, "A Jacobi operational matrix for solving a fuzzy linear fractional differential equation," Advances in Difference Equations, vol. 104, no. 1, pp. 1-29, 2013.

[10] Z. J. Fu, W. Chen, and H. T. Yang, "Boundary particle method for Laplace transformed time fractional diffusion equations," Journal of Computational Physics, vol. 235, pp. 52-66, 2013.

[11] G. F. Pang, W. Chen, and Z. J. Fu, "Space-fractional advectiondispersion equations by the Kansa method," Journal of Computational Physics, vol. 293, pp. 280-296, 2015.

[12] A. H. Bhrawy and M. A. Zaky, "A method based on the Jacobi tau approximation for solving multi-term time-space fractional partial differential equations," Journal of Computational Physics, vol. 281, pp. 876-895, 2015.

[13] A. H. Bhrawy, M. M. Alghamdi, and T. M. Taha, "A new modified generalized Laguerre operational matrix of fractional integration for solving fractional differential equations on the half line," Advances in Difference Equations, vol. 179, no. 1, pp. 1-12, 2012.

[14] A. H. Bhrawy and A. S. Alofi, "The operational matrix of fractional integration for shifted Chebyshev polynomials," Applied Mathematics Letters, vol. 26, no. 1, pp. 25-31, 2013.

[15] S. Shen, F. Liu, J. Chen, I. Turner, and V. Anh, "Numerical techniques for the variable order time fractional diffusion equation," Applied Mathematics and Computation, vol. 218, no. 22, pp. 10861-10870, 2012.

[16] Y. M. Chen, L. Q. Liu, B. F. Li, and Y. Sun, "Numerical solution for the variable order linear cable equation with Bernstein polynomials," Applied Mathematics and Computation, vol. 238, pp. 329-341, 2014.

[17] Y. M. Chen, L. Q. Liu, X. Li, and Y. Sun, "Numerical solution for the variable order time fractional diffusion equation with Bernstein polynomials," CMES. Computer Modeling in Engineering \& Sciences, vol. 97, no. 1, pp. 81-100, 2014.

[18] S. Chen, F. Liu, and K. Burrage, "Numerical simulation of a new two-dimensional variable-order fractional percolation equation in non-homogeneous porous media," Computers \& Mathematics with Applications, vol. 68, no. 12, pp. 2133-2141, 2014.

[19] P. Zhuang, F. Liu, V. Anh, and I. Turner, "Numerical methods for the variable-order fractional advection-diffusion equation with a nonlinear source term," SIAM Journal on Numerical Analysis, vol. 47, no. 3, pp. 1760-1781, 2009.

[20] L. F. Wang, Y. P. Ma, and Y. Q. Yang, "Legendre polynomials method for solving a class of variable order fractional differential equation," Computer Modeling in Engineering \& Sciences, vol. 101, no. 2, pp. 97-111, 2014. 
[21] Y.-M. Chen, Y.-Q. Wei, D.-Y. Liu, and H. Yu, "Numerical solution for a class of nonlinear variable order fractional differential equations with Legendre wavelets," Applied Mathematics Letters, vol. 46, pp. 83-88, 2015.

[22] Q. Y. Li, N. C. Wang, and D. Y. Yi, Numerical Analysis, Tsinghua University Press, Beijing, China, 2008. 


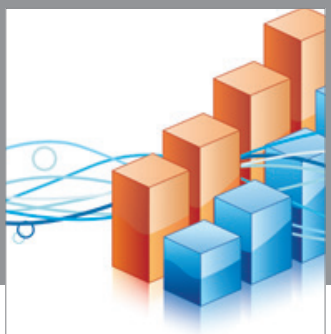

Advances in

Operations Research

vatem alat4

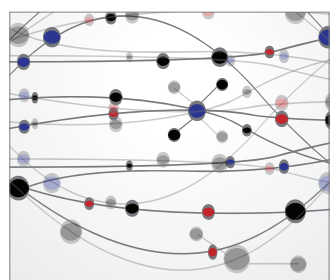

\section{The Scientific} World Journal
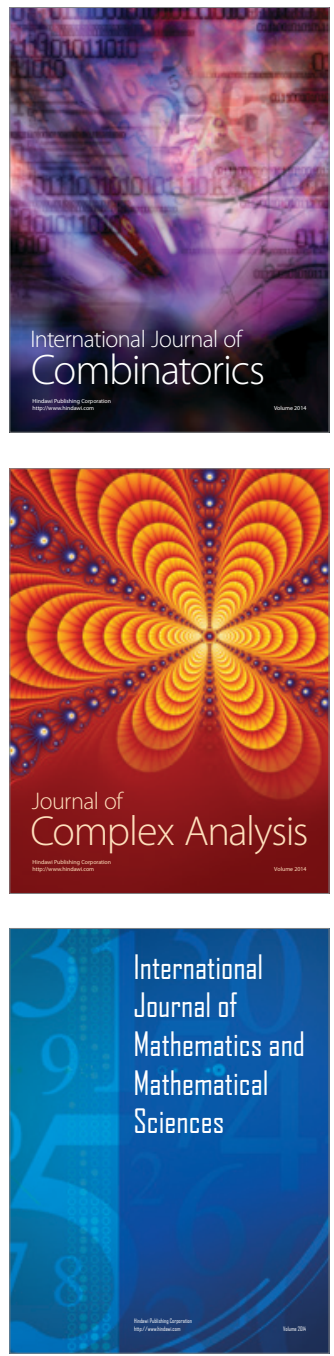
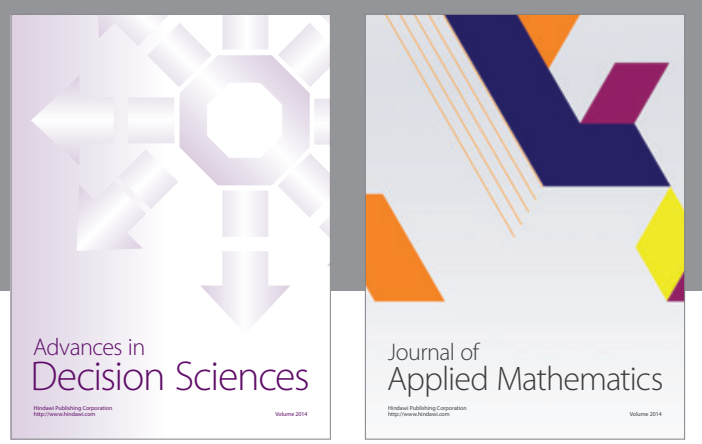

Algebra

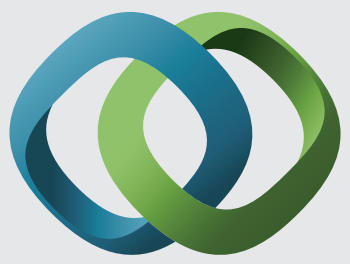

\section{Hindawi}

Submit your manuscripts at

http://www.hindawi.com
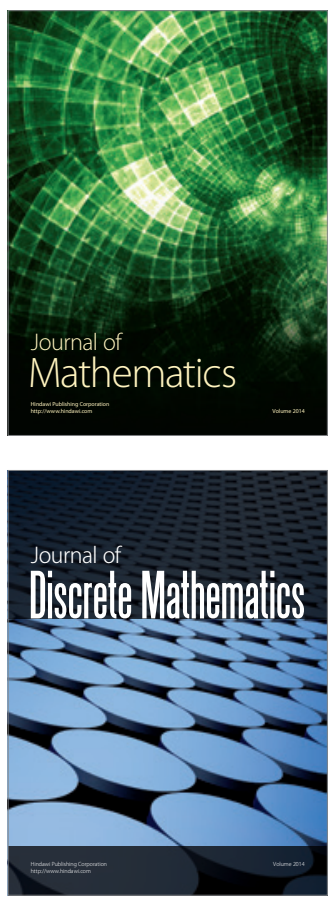

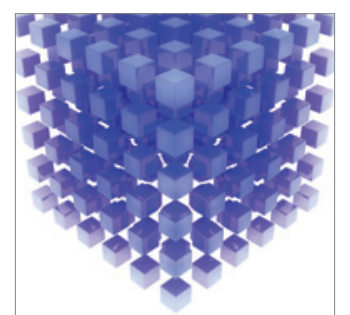

Mathematical Problems in Engineering
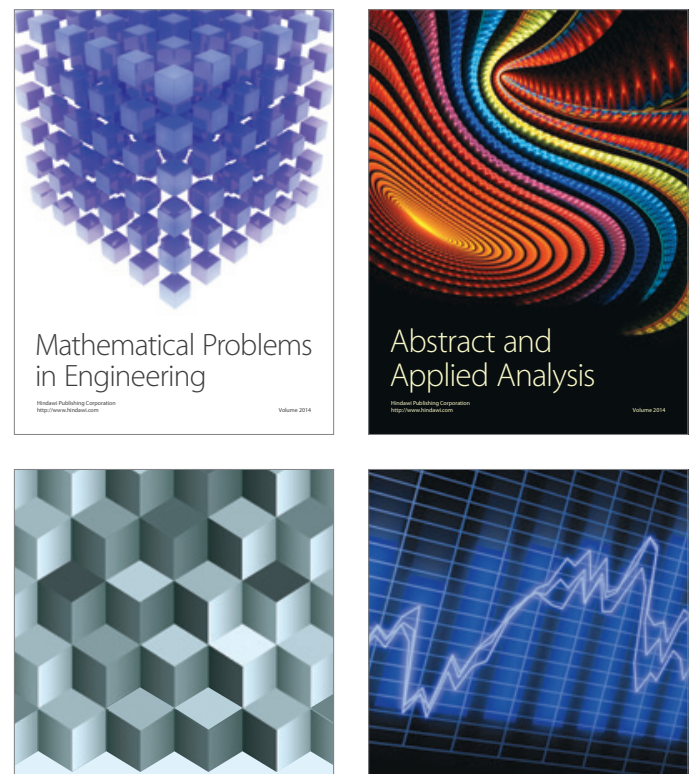

Journal of

Function Spaces

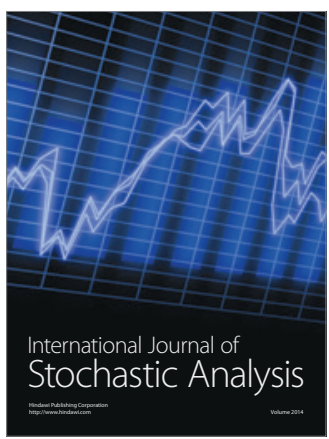

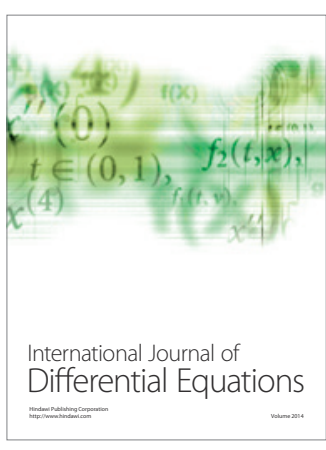
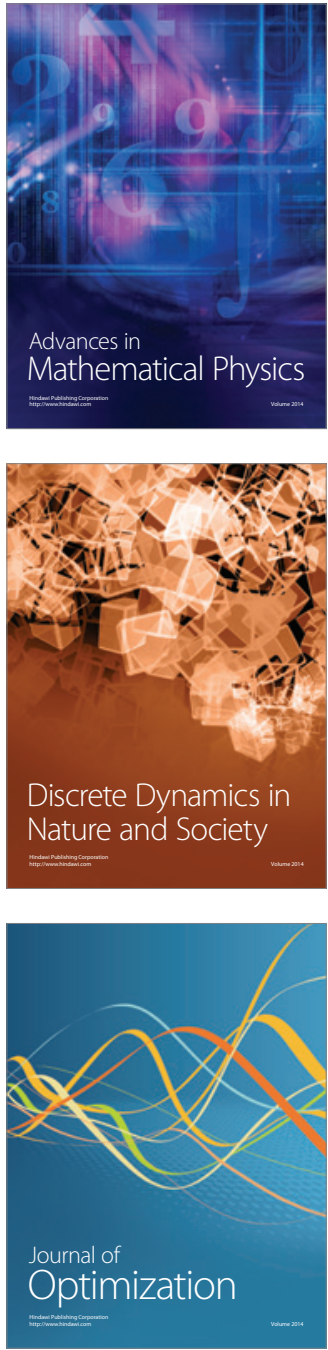